\title{
Fullwave Doppler Reflectometry Simulations for Density Turbulence Spectra in ASDEX Upgrade Using GENE and IPF-FD3D
}

\author{
C Lechte ${ }^{1}$, GD Conway ${ }^{2}$, T Görler ${ }^{2}$, T Happel ${ }^{2}$ and the \\ ASDEX Upgrade Team ${ }^{2} \ddagger$ \\ ${ }^{1}$ Institute of Interfacial Process Engineering and Plasma Technology IGVP, \\ Universität Stuttgart, 70569 Stuttgart, Germany \\ ${ }^{2}$ Max-Planck-Institute for Plasma Physics, 85748 Garching, Germany \\ E-mail: carsten.lechte@igvp.uni-stuttgart.de
}

(Some figures may appear in colour only in the online journal )

\begin{abstract}
Doppler reflectometry is an important microwave diagnostic for turbulent fusion plasmas. A microwave beam is scattered off density fluctuations, with wavenumbers according to incident frequency and relative direction. This way, a density fluctuation spectrum is measured. However, the correspondence between fluctuation power and scattered microwave power is strongly non-linear. Studying these processes numerically therefore requires fullwave simulations (IPFFD3D), with density fluctuations supplied by a separate plasma turbulence code (GENE). Earlier such attempts suffered from the low number of data points in the "interesting" part of the spectrum, where a power law had to be fitted to 4 or 5 points. This has been overcome with recent simulations, which now show a much better agreement with experimental data.
\end{abstract}

Keywords: plasma diagnostics, Doppler reflectometry, simulation, microwaves

Submitted to: Plasma Sci. Technol.

\section{Introduction}

Magnetically confined fusion plasmas are usually plagued by instabilities which result in turbulent fluctuations of important plasma parameters. Turbulence is a driver of particle and energy transport out of the magnetic confinement. Reflectometry methods are widely used to measure density fluctuations inside the plasma, which can have temperatures of several $\mathrm{keV}$.

Doppler reflectometry (or Doppler backscattering) is an important microwave diagnostic for turbulent fusion plasmas. A microwave beam is reflected at the cutoff and scattered off density fluctuations, with wavenumbers according to incident frequency and relative direction. The direct (0th order) reflection is ignored, and usually the -1 st Bragg order is the desired signal. It propagates backwards through

$\ddagger$ see the author list of H. Meyer et al. 2019 Nucl. Fusion 59112014 
the same plasma region as the incident beam, illustrated in figure 1. For a monostatic antenna configuration the Bragg condition for the received scattered radiation is:

$$
\vec{k}_{\text {fluct }}=-2 \vec{k}_{i}=-2 \vec{N} k_{o}
$$

where $\vec{k}_{\text {fluct }}$ is the probed density fluctuation wavenumber, $\vec{k}_{i}$ the incident beam wavevector at the turning point and $k_{o}$ the microwave vacuum wavenumber. $\vec{k}_{f l u c t} \equiv$ $\vec{k}_{\perp}$ is perpendicular both to the background magnetic field and the radial direction. For a slab geometry, i.e. flat refractive index layers, $N=\sin \theta$ where $\theta$ is the angle between the incident wave and the flux surface normal at the vacuum-plasma interface, i.e. the radial direction. However, for the general case of curved flux/refractive index surfaces it is necessary to use ray or beam tracing techniques to determine $N^{2}=N_{\perp}^{2}+N_{\|}^{2}[1]$.

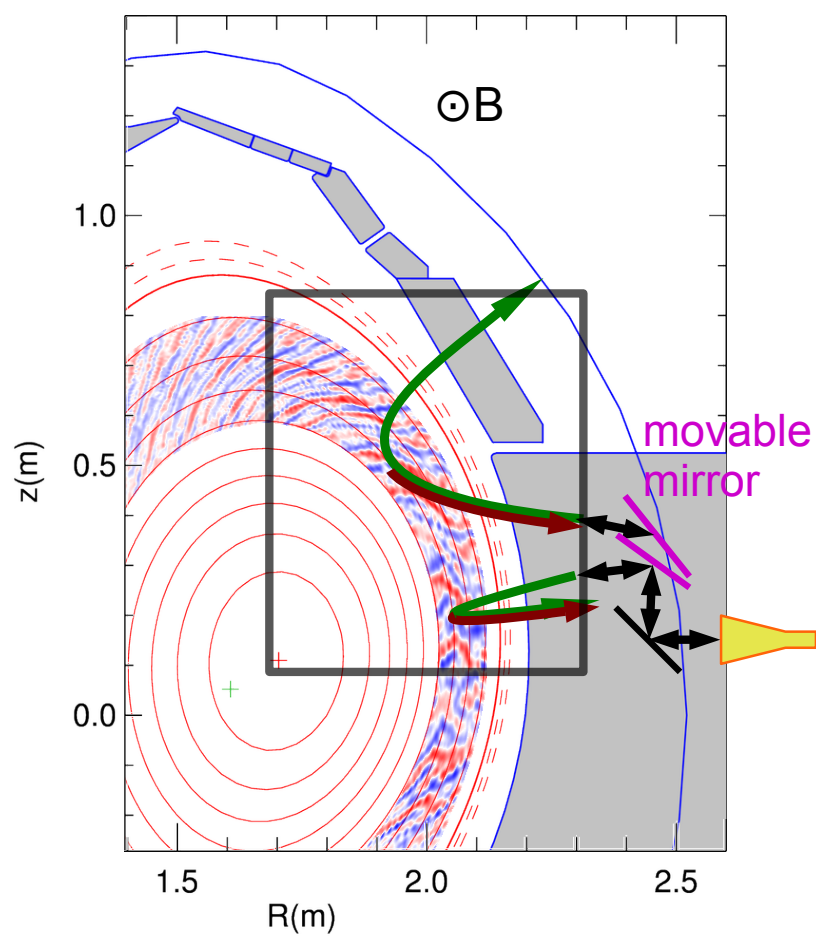

Figure 1. A vertical cross section of the ASDEX Upgrade fusion experiment shows the sketch of the monostatic antenna for Doppler reflectometry, with 2 steering mirror positions corresponding to 2 beam paths. The input beams are shown in green, the back-scattered beam in red. The filled contours show the density field from a turbulence simulation. The red lines are the magnetic flux surfaces. The simulation region is marked with a black frame.

A density fluctuation wavenumber spectrum can be measured by varying $k_{\text {fluct }}$. It is usually assumed that the main contribution to the scattering signal is concentrated to a small radial region in front of the cutoff layer. However, the correspondence between fluctuation power and scattered microwave power is strongly non-linear and must be investigated with appropriate methods. In this paper, we employ fullwave simulations using the code IPF-FD3D, with density fluctuations supplied by a separate plasma turbulence code (GENE). Earlier fullwave simulations suffered from the low number of data points in the interesting part of the spectrum, where a power law had 
to be fitted to 4 or 5 points. This has been overcome with recent simulations, which now show a much better absolute agreement with experimental data.

Since a fusion plasma with an external magnetic field is not isotropic for electromagnetic waves, there exist 2 propagation modes: In $\mathrm{O}$ (ordinary) mode, the electric field of the wave is parallel to the background magnetic field lines. In $\mathrm{X}$ (extraordinary) mode, the gyromotion of the electrons interacts with the wave electric field, which is perpendicular to the background magnetic field. The two propagation modes have different dispersion relations, and different cutoff frequencies that can depend on the magnetic field strength. The same fluctuations measured in $\mathrm{O}$ and $\mathrm{X}$ mode can give spectra that are very different from each other, and different again to the underlying density fluctuation spectrum. This has long been a point of contention when comparing experimental Doppler measurements of the spectrum with plasma turbulence codes and was resolved by implementing a synthetic diagnostic that accurately models the Doppler reflectometry or backscattering diagnostic $[2,3,4]$ for ASDEX Upgrade. The same approach, reported in [5], has been taken for the FT-2 tokamak using both the fullwave code and a fast linear method for the synthetic diagnostic, with the ELMFIRE full-f gyrokinetic code providing the plasma turbulence.

While there are many degrees of freedom in a fluctuation spectrum, there are two parameters that are typically used to describe a turbulence spectral shape, a transition wavenumber $k_{t}$ ("knee"), below which the spectrum is shallow, and an exponent $n$ that describes the decay of the spectrum for $S\left(k_{\perp}>k_{t}\right) \propto k_{\perp}^{n}$. Typical numbers for $n$, also called the spectral index, are $-5 / 3$ and -3 for three- and two-dimensional neutral fluid turbulence $[6,7]$. For plasma turbulence, measured values for $n$ range from -2 to -9 . In fusion plasmas, the spatial scale is often normalised by the drift scale $\rho_{s}=c_{s} / \omega_{c i}$, where $c_{s}$ is the local ion sound speed and $\omega_{c i}$ is the local ion cyclotron frequency. With this scaling, the most unstable wavenumber for ion temperature gradient (ITG) turbulence given by $k_{\perp} \rho_{s} \approx 0.3$.

Sophisticated plasma turbulence simulation codes, such as GENE [8, 9] are used to investigate the plasma turbulence. Since they output the plasma quantities (density, temperature etc.) directly, a one-to-one comparison to the output of plasma diagnostics neglects any influence of the diagnostic system. Therefore, a synthetic diagnostic is implemented using the fullwave code IPF-FD3D for the diagnostic part, coupled to the GENE code which supplies the turbulent density fields.

\section{The Synthetic Diagnostic}

Figure 2 illustrates the problem when comparing turbulence simulations directly to experimental measurements. Unless the diagnostic delivers the same physical quantity as the simulation, it is possible that the measurement system biases the data. Both $\mathrm{O}$ and $\mathrm{X}$ mode measured the same turbulence, but yield spectra that differ significantly in their two classification parameters: the knee positions are different (black vs. red vertical line), and the spectral indices on either side are different. The knee of the GENE simulations coincides with the X mode knee, but the X mode spectrum is nearly flat at low wavenumbers, whereas the GENE spectrum still has a strong slope. The position of the knee is tied to the underlying instability that drives the turbulence, with ion temperature gradient (ITG) modes being dominant on the left side and electron temperature gradient (ETG) modes being dominant on the right of the knee. It would seem that $\mathrm{O}$ mode reflectometry measures a plasma with a different drive. 


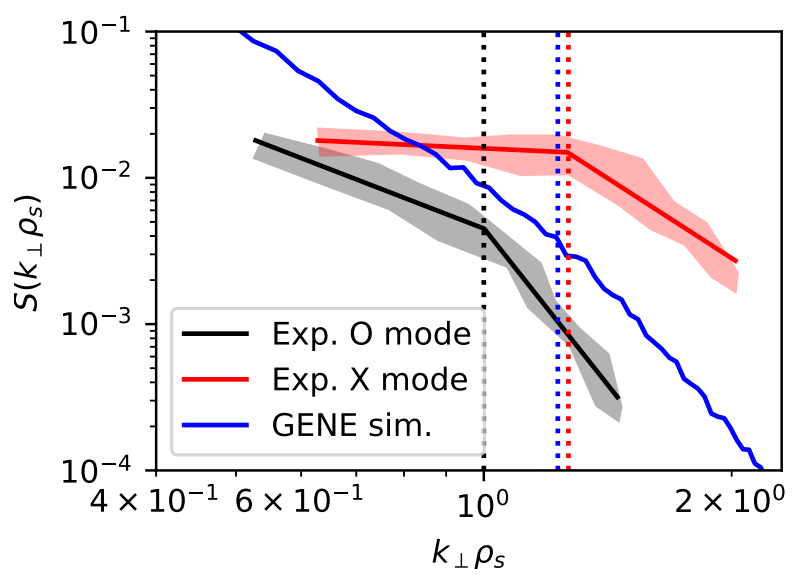

Figure 2. The problem: Directly comparing experiment with simulation output yields a large discrepancy. Power spectrum of the density fluctuations over the normalised wavenumber, all taken at the same radial position $\rho_{\text {pol }}=0.825$. Exp: backscattered microwave power (AUG shots \#31259,60,64,66), GENE simulation: direct density fluctuations. The vertical lines mark the knee positions of the three spectra. Data previously published in [4].

It is therefore necessary to also simulate the plasma diagnostic and then compare like with like. This synthetic diagnostic takes into account both the physical system (turbulence code) and the plasma diagnostic (fullwave code), which takes the turbulence simulations as input and calculates the signal seen by the experimental Doppler reflectometer. We expect a good agreement between exerimental and synthetic signals for $\mathrm{O}$ and $\mathrm{X}$ mode, respectively. The significant difference of both experimental and synthetic spectra to the GENE spectrum are then explained by the characteristic instrument function of the Doppler diagnostic. One can then speculate how to recover the true fluctuation spectrum from measurements without the turbulence and fullwave simulations. If experimental and fullwave spectra agree, this also validates the turbulence code [3].

A considerable part of the effort for this synthetic diagnostic has been dedicated to generating the physically consistent turbulence fields for its input. The code used was the gyrokinetic plasma turbulence code GENE $[8,9]$ which is a physically comprehensive, hyperscalable Vlasov code that supports both local (flux-tube) and global (full-torus) calculations. However, as the latter can be quite computationally demanding, a local flux tube around the toroidal flux radius probed in experiment was simulated, with physics inputs being interpolated from the measured background profiles. The same profiles were used for the fullwave code IPF-FD3D, with the added turbulence from GENE. In order to ensure the relevance of the turbulence simulations, the global heat flux of the simulation was matched to the flux measured in the experimental discharges that were modelled. This measurement is independent of the Doppler reflectometry diagnostic. The temperature profiles were then adjusted within the experimental uncertainty until the experimental flux was matched by the simulations. The procedure is described in more detail in references $[2,4]$. 


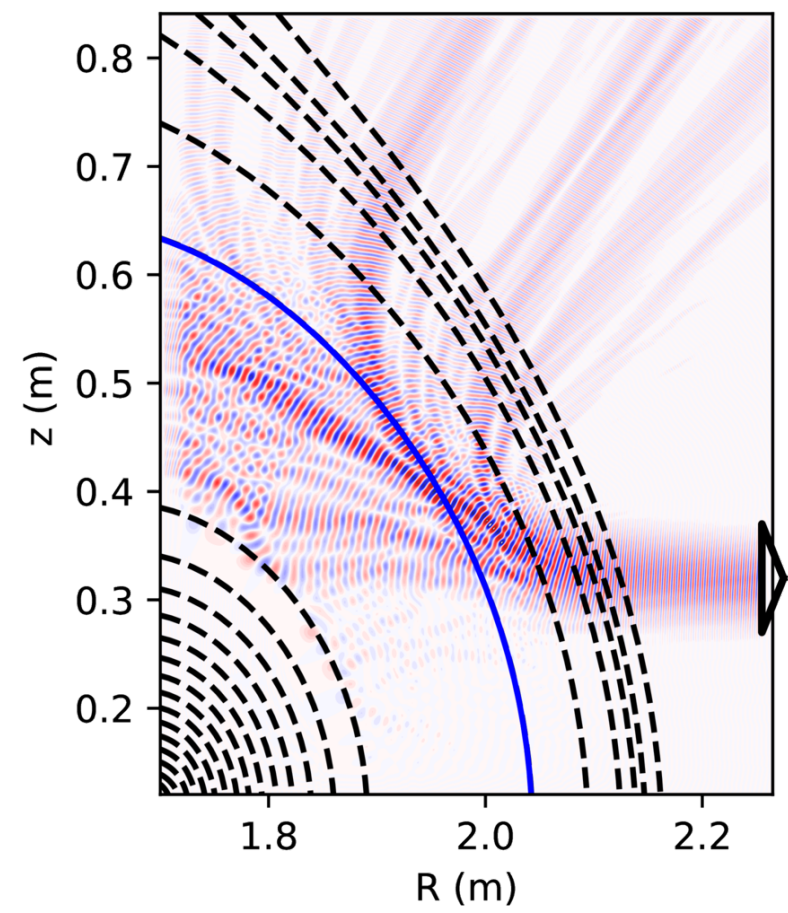

Figure 3. Setup of the simulation grid for ASDEX Upgrade. The electric wave field of a single beam is shown as filled contours overlaid by the dashed line contour plot of the background density profile with the cutoff position at $\rho_{\text {pol }}=0.825$ as solid blue line. The virtual sending and receiving antenna is indicated at the lower right edge.

\section{The Fullwave Code IPF-FD3D}

The reflectometry simulations were performed with the IPF-FD3D code $[10,11]$ using a two-dimensional Cartesian grid. The wave propagation is simulated from the launching antenna, to the plasma cutoff, and back to the coherent receiver, yielding the instantaneous reflectometer amplitude and phase. IPF-FD3D is a finite-difference time domain (FDTD) code [12] that simulates the propagation of electromagnetic waves in cold plasmas $[13,14]$ in two or three dimensions. Its main feature is the capability of simulating wave propagation in a given inhomogeneous, anisotropic (i.e. magnetized) plasma by integrating the equation of motion of the electrons and the Maxwell equations:

$$
\begin{aligned}
\frac{\partial}{\partial t} \mathbf{E} & =\frac{1}{\varepsilon_{0}} \nabla \times \mathbf{H}-\frac{1}{\varepsilon_{0}} \mathbf{J} \\
\frac{\partial}{\partial t} \mathbf{J} & =\varepsilon_{0} \omega_{p e}^{2} \mathbf{E}-\omega_{c e} \mathbf{J} \times \widehat{\mathbf{B}}_{0}
\end{aligned}
$$




$$
\frac{\partial}{\partial t} \mathbf{H}=-\frac{1}{\mu_{0}} \nabla \times \mathbf{E}
$$

where $\mathbf{E}$ and $\mathbf{H}$ are the electric and magnetic field vectors of the wave, and $\mathbf{J}$ denotes the vector for the plasma current caused by the wave. Furthermore, $\widehat{\mathbf{B}}_{0}$ is the unity vector in the direction of the background magnetic field $\mathbf{B}_{0}, \omega_{c e}$ the electron cyclotron frequency, and $\omega_{p e}$ the plasma frequency. All these quantities can be time and space dependent.

The 3 differential equations are converted to finite difference equations on a staggered Yee grid in space and leap-frogging of $\mathbf{E}, \mathbf{H}$, and $\mathbf{J}$ in time [15]. This allows the use of central differences with second order accuracy. Equation (3) presents a problem with the choice of the time grid because it contains both $\mathbf{J}$ and its time derivative. This is overcome by interpolating the required values of $\mathbf{J}$ between the available time points with a Crank-Nicolson like formulation, which, however, does not give unconditional stability like it does for parabolic problems.

The plasma setup is given by spatial arrays of plasma density and the three background field components $B_{r}$ (radial), $B_{t}$ (toroidal), and $B_{p}$ (poloidal). They are taken from the ASDEX Upgrade reconstructed equilibria (prepared) by CLISTE [16] for each experimental shot that is considered in the simulations. The density fluctuations from the GENE simulations are added on top of the equilibrium density profile, with an optional scaling factor to artificially increase or decrease the fluctuation amplitude. Typical amplitudes were on the order of 1-3 percent of the background density. All simulations reported here were done in $2 \mathrm{D}$, i.e. in the poloidal $R-z$ plane.

The reflectometer launching and receiving antennas are synthetic apertures at the edge of the simulation grid. The beams are fundamental Gaussians that can be modified by higher order Hermite modes so that the experimental beam geometry can be accurately modelled. In this paper, the beams were modelled as pure Gaussian beams with a beam waist corresponding to the experimental beam widths. The receiver samples the field at the same position and convolves it with the complex beam pattern of the receiving antenna, which is usually the same as the launching antenna. Figure 3 shows a simulation grid which covers the upper right portion of the poloidal plasma cross section of ASDEX Upgrade. Overlaid on the density contour plot is the electric wave field, which is injected in the upper right edge. The many backscattered waves are seen in the lower half. The code uses a total/scattered field format to discriminate the weak scattered signal from the input wave.

For the Doppler reflectometry investigation a radial position $\rho_{\text {pol }}$ is chosen, which is based on the poloidal magnetic flux. The beam tracing code TORBEAM [17] is used to find the corresponding launch frequencies and their probed wavenumbers $k_{\perp}$ for a range of antenna tilt angles. All beams are launched into the simulation grid simultaneously. They are discriminated by their different frequencies, with the relative cross talk between beams below $10^{-10}$. While all beams have slightly different propagation angles, it is not possible to discriminate by that, since the wavenumber spectra of the beams overlap significantly. This receiver part of the code was recently speeded up by a factor of 80 , which allows an increase of simultaneously launched beams from less than 20 to $160-240$.

An animation illustrating the Doppler backscattering process is available [18]. 

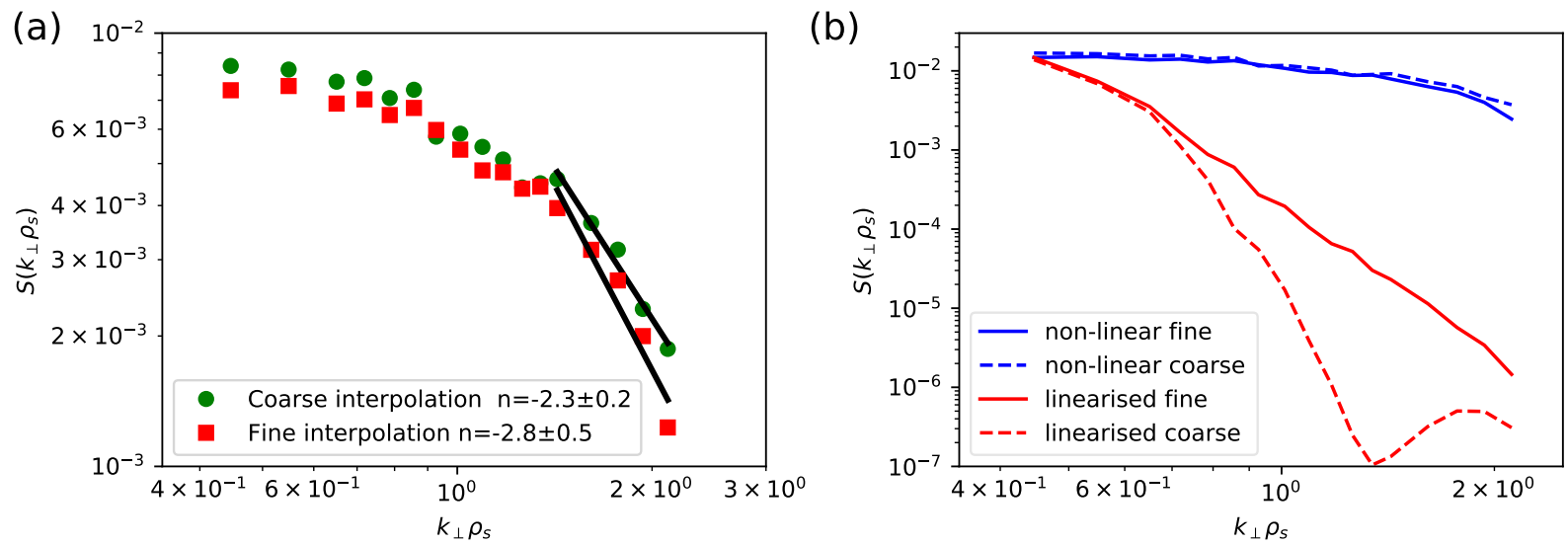

Figure 4. Comparing fine and coarse resolution of the interpolation grid for the turbulence data in X mode. Left: Power law fits, the spectral index changes but only within its large error bars. Right: Loss of spectral power at large wavenumbers due to coarse interpolation is masked in the non-linear case. This becomes apparent at 1/1000 of the density fluctuation amplitude, where nonlinear effects vanish. "Coarse" data taken from [2].

(a)

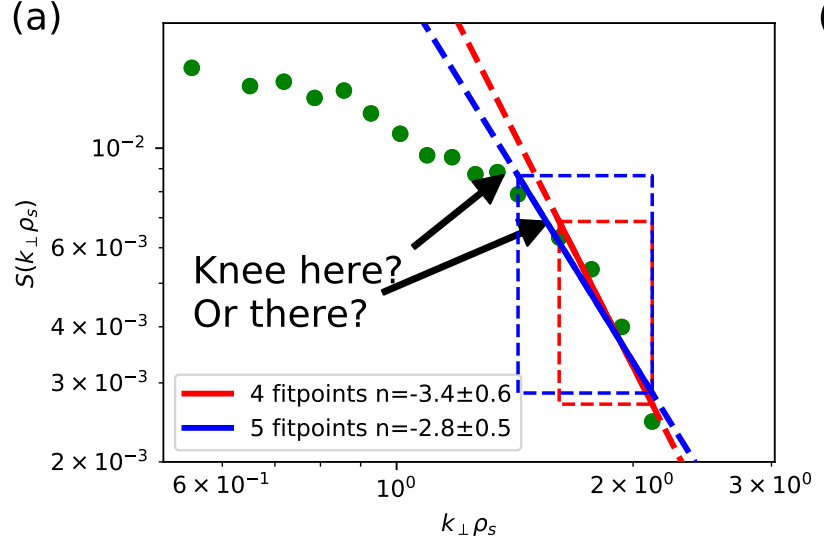

(b)

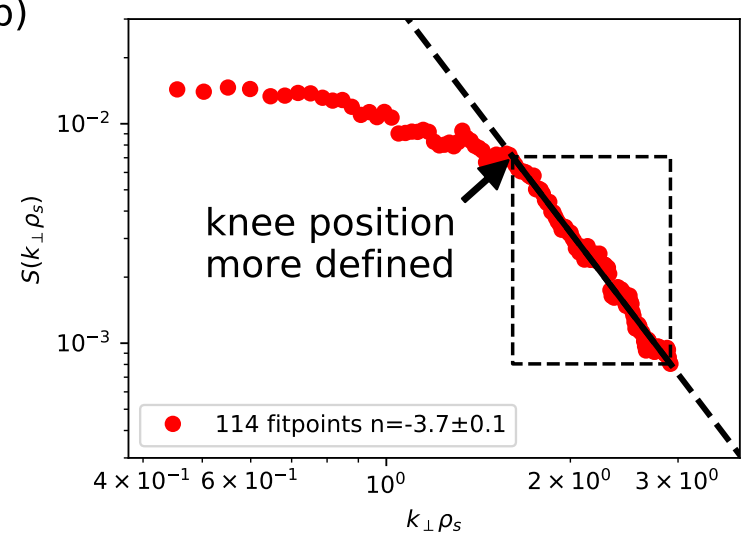

Figure 5. Left: Different power law fits to the very few points in the Doppler spectrum from previous fullwave simulations [4]. Right: New high-resolution simulated Doppler spectrum with obvious knee position and robust fit to power law.

\section{Results of The Doppler Reflectometry Simulations}

\subsection{Previous Results}

Previous results for ASDEX Upgrade are discussed in [2] and, with more recent experimental data in both $\mathrm{O}$ and $\mathrm{X}$ mode, in [4]. The new results concern only the measurement campaign in the latter publication (AUG shots \#31259,60,64,66). The main result was that in $\mathrm{X}$ mode, the spectrum saturates at low wavenumbers 
(where the spectral power is highest). The overall relative fluctuation strength was $0.9 \%$, which is given by the GENE simulations. It is the value that reproduces the experimentally observed (electron) heat flux. This saturation changes the apparent location of the knee, where the spectrum goes from very shallow to the steep power law region at large wavenumbers. The power law exponent (spectral index) is also modified, the spectrum is more shallow ( $n \approx-2.3$ instead of $n \approx-3.6$ and $n \approx-6.3$ at high wavenumbers). This is an effect both of the non-linear compression of the spectrum, and non-linear enhancement at intermediate fluctuation amplitudes first observed in [19]. Figure 4 (right) illustrates the significant difference between a spectrum measured at non-linear vs. linear conditions (compare only the solid curves labelled "fine").

When the density fluctuation amplitude is decreased to $1 / 2$, the non-linear compression effect is reduced and the spectrum steepens again. With this factor, the spectrum from fullwave simulations of GENE data, and the ones from experimental data, are in better agreement. The scaling factor was justified by the significant uncertainty in the ion temperature profiles that were used as input to GENE, which are the drivers of the turbulence. In $\mathrm{O}$ mode probing, the degree of non-linearity was much lower, and the fullwave and experimental spectra were more similar.

This situation was not satisfactory, especially the power law fits to only 4 or 5 points, with significant differences in spectral index between the two, albeit with their respective uncertainties, which were large $(>25 \%)$. One cannot justify from the data alone which fit is the correct one. This is shown in figure 5 (left). Therefore, 2 improvements were made. Only the results of these improvements and comparison with experimental data from AUG shots \#31259,60,64,66 and GENE results will be discussed in the rest of the paper:

(i) The GENE turbulent density fields were re-exported from GENE with an 8 times finer interpolation grid in the direction along $B_{0}$, and

(ii) the number of points in the spectrum, previously the exact same ones that were measured experimentally, was increased to 160 for X mode and 220 for $\mathrm{O}$ mode. This was only feasible because of the optimisations described in section 3 .

Regarding point (i) The GENE turbulence code works in coordinates that are aligned to the magnetic background field. When the plasma density is exported to IPF-FD3D, it must be interpolated onto the Cartesian grid used in IPF-FD3D. It was discovered that the interpolation along the magnetic field direction was using too few points. This had the effect of suppressing certain wavenumbers in the perpendicular direction, which the Doppler reflectometry is measuring. This was not immediately obvious, because of the non-linear effect of higher order scattering, where the high amplitude, low wavenumbers cause Doppler scattering at twice their wavenumber, filling the gap in the spectrum. At full density fluctuation amplitude, there is only a small difference in the Doppler spectrum between the old coarse and the new, much finer interpolation (see figure 4 (left)). While the spectral index does change by more than $20 \%$, this is still within the uncertainty ranges. The change is going in the direction of the experimental value of $n=-3.6$. In figure 4 (right), the density fluctuation amplitude has been scaled by $1 / 1000$ before running the fullwave code. This linearised case shows the significant loss of spectral power in the coarse interpolation compared to the fine one. 


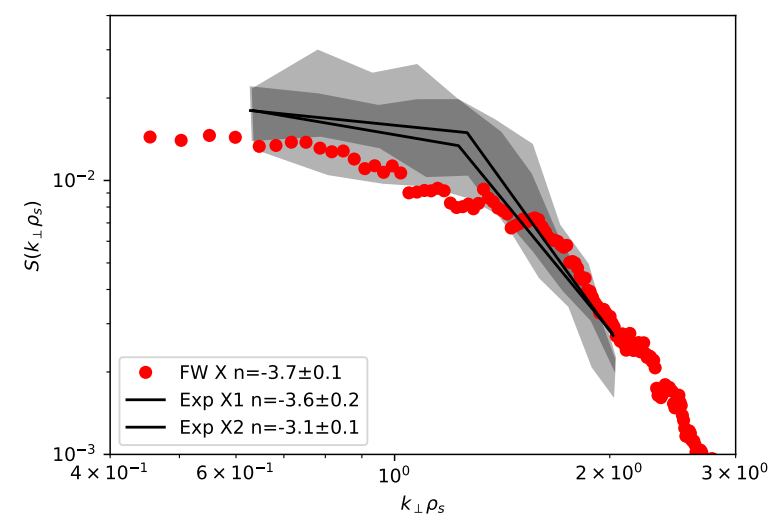

Figure 6. New fullwave simulation X mode Doppler spectra ("FW", red circles) and overlaid envelope of experimental results from AUG shots \#31259,60,64,66. Experimental results were already reported in [4]. The spectral power in arbitrary units was scaled to achieve maximum overlap between experiment and simulation.

Regarding point (ii) The new fullwave simulations used the fine interpolation of the turbulence data and increased the number of probed wavenumbers, which is not feasible in experiment. In X mode simulations, for example, the launched frequencies ranged from 98.3 to $106.1 \mathrm{GHz}$ on a fixed raster of $50 \mathrm{MHz}$. The resulting simulated Doppler spectrum is shown in figure 5 (right). The knee position in the spectrum is now very clearly identified, and the power law fit to 114 points produces a robust result of $n=-3.7 \pm 0.1$ with a low error estimate. We are now confident that the match with the experimental spectral index is not accidental. No scaling factors for the turbulence strength are necessary to achieve the same spectral index $n$ as in experiment, which is caused by underlying fluctuations with $n \approx-6.3$.

\subsection{Results for $X$ Mode}

Figure 6 overlays the $\mathrm{X}$ mode spectra from simulation and the envelope of two different measurements. The solid black lines are fits to the measured data points. The fullwave simulation covers a larger wavenumber range than possible in the experiment. Since there is no absolute power calibration, the experimental spectrum has been scaled to fit all fullwave points into the envelope. The spectral index now coincides with one of the measurements, and is still similar to the other measurement. The position of the knee is still at lower wavenumbers in the measurements. This can partially be explained by uncertainties in the determination of the wavenumber using beamtracing [20]. Overall, a good agreement has now been reached between experiment and simulation.

Finally, the scaling behaviour was analysed by artificiallylowering the fluctuation amplitude until the non-linear effects vanished. Let $\mathfrak{F}(\cdot)$ denote the function that yields the wavenumber power spectrum at some level of turbulence scaling. $\mathfrak{F}(1)$ is the spectrum shown in figure 6 with the unscaled GENE turbulence, $\mathfrak{F}(1 / 10)$ is the spectrum obtained when the density flucutation amplitude is divided by 10 before performing the fullwave simulations. In a fully linear case, $\mathfrak{F}(1)=S^{2} \mathfrak{F}(1 / S)$ with a scaling factor $S \geq 1$. In the non-linear case, a saturated spectrum has 


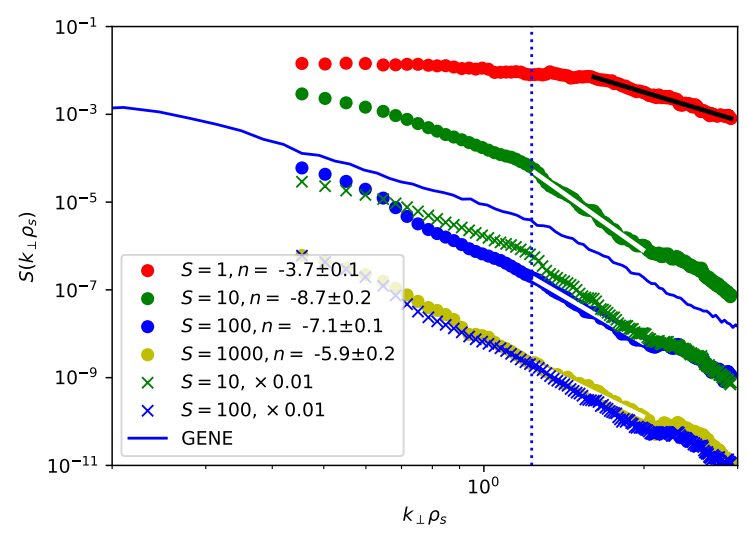

Figure 7. Doppler spectra from $\mathrm{X}$ mode scattering of scaled turbulence amplitude $(\bullet)$ and 2 overlaid scaled spectra $(x)$. The GENE spectrum is shown for comparison, with the vertical line marking its knee position. The wavenumber ranges for the spectral index fit are indicated by the solid white and black lines. The unscaled spectrum " $\mathrm{S}=1$ " is the same as in figure 6

$\mathfrak{F}(1) \ll 100 \cdot \mathfrak{F}(1 / 10)$.

The results are shown in figure 7 . The circles show the Doppler spectrum for turbulence amplitudes scaled for $S=1,10,100$, and 1000. One can see the apparent knee move from its position near normalised wavenumber 1.6 to 1.3, according to the lessened effect of saturation. The knee actually coincides with the knee of the GENE spectrum. However, for $S \geq 100$, the spectrum does not have a prominent knee. The biggest shape change is between unscaled turbulence and 1/10 amplitude. (The wavenumber ranges for the spectral index fits have been adapted for the different values of $S$.) We find $\frac{1}{100} \mathfrak{F}(1 / 100) \approx \mathfrak{F}(1 / 1000)$. The left hand side of this expression is plotted as blue crosses, and the right hand side plotted as yellow circles. We conclude that at turbulence amplitudes of $1 / 100$ the original $0.9 \%$, the Doppler scattering process becomes linear.

The degree of non-linearity can be estimated using a parameter introduced in [21]:

$$
\gamma=\left(\frac{\delta n}{n}\right)^{2} \frac{G^{2} \omega^{2} x_{c} l_{c x}}{c^{2}} \ln \frac{x_{c}}{l_{c x}}
$$

where $\frac{\delta n}{n}$ is the turbulene level, $G$ is a polarisation dependent factor, $\omega$ is the probing angular frequency, $x_{c}$ is the distance from edge to cutoff, $l_{c x}$ is the radial correlation length, and $c$ is the speed of light. It indicates non-linearity if $\gamma \gg 1$, and a linear regime if $\gamma \ll 1$. Its value decreases from $\gamma=82$ [4] for the unscaled turbulence (green circles) to $\gamma=0.0082$ for the $1 / 100$ scaled turbulence (blue circles), in agreement with the spectrum going from non-linear to linear.

Furthermore it can be seen that an underlying spectrum of density fluctuations from GENE with a spectral index of $n=-6.3$ is measured in the linear cases with a slightly larger spectral index $(n \approx-7)$, which is due to the reduced sensitivity of the Doppler reflectometry diagnostic at large wavenumbers. This phenomenon was reported e.g. in $[10,19]$. 


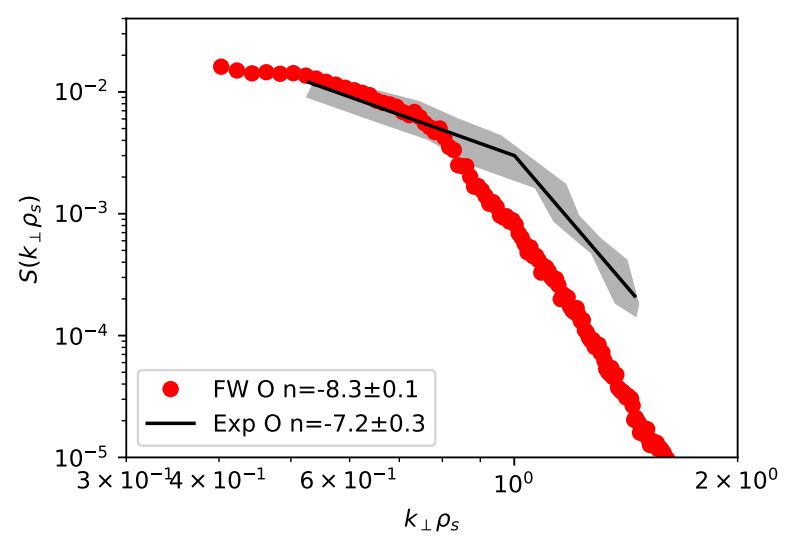

Figure 8. New fullwave simulation O mode Doppler spectra ("FW", red circles) and overlaid envelope of experimental results from AUG shots \#31259,60,64,66. Experimental results were already reported in [4].

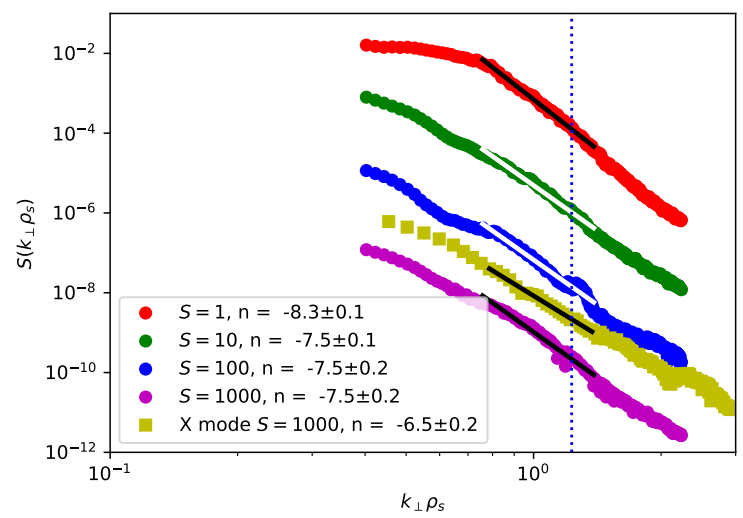

Figure 9. Doppler spectra from $\mathrm{O}$ mode scattering of scaled turbulence amplitude ( ) and linearised X mode for comparison. The wavenumber ranges for the spectral index fit are indicated by the solid white and black lines. The unscaled spectrum " $\mathrm{S}=1$ " is the same as in figure 8. The GENE spectrum knee position is marked with the vertical line.

\subsection{Results for $O$ Mode}

The results for $\mathrm{O}$ mode reflectometry are shown in figure 8 . As in the $\mathrm{X}$ mode results, the knee is at a different position, but the spectral index is nearly the same. However, the shift of the knee is in the opposite direction compared to the $\mathrm{X}$ mode results. Further investigations are necessary to resolve this contradiction. At low wavenumbers, the $\mathrm{O}$ mode spectra are not compressed into a flat plateau. It is therefore expected that the $\mathrm{O}$ mode measurements are less non-linearly saturated than X mode. This is born out by a $\gamma$ value of approximately 2 . 
The scaling treatment for $\mathrm{O}$ mode is shown in figure 9. As expected from the $\gamma$ value, a linear situation was reached already for $S=10$, which confirms that O mode measurements of the same turbulence are less advanced into non-linear saturation. The knee moves to lower wavenumbers again for $S=10$. For larger values of $S$, the spectrum develops several bumps and the spectral index becomes larger (less steep) in the original wavenumber range.

For comparison, the linearised X mode spectrum is plotted. It is quite similar to the linearised $\mathrm{O}$ mode spectrum, albeit less steep in the fit range, which is at lower wavenumbers than in $\mathrm{X}$ mode.

\subsection{Discussion}

In comparison of fullwave simulation and experiment, the knee and the whole spectrum seems to be shifted in wavenumber by $25 \%$ in the opposite direction compared to $\mathrm{X}$ mode. However, the shape of the spectrum given by the spectral indices is reproduced well. This is an important improvement over the previous results, where the spectral indices were less well defined.

Possible errors in determining the probed wavenumber from beam or raytracing are investigated in [20]. An additional cause for discrepancies is the $3 \mathrm{D}$ nature of the experiment and TORBEAM, which is not fully modelled in the 2D fullwave simulations. It is therefore planned to run additional fullwave simulations with marker peaks in the spectrum to determine the actual probed wavenumbers in the $2 \mathrm{D}$ scenario modelled in the fullwave code.

Furthermore, a more sophisticated analysis of non-linearity thresholds and apparent knee positions can be performed using the theoretical framework given in [22]. For a preliminary test, we are only looking at the exponential term in the formula for the received signal power (adapted from eq. (19) in [22]):

$$
\left\langle\left|A_{s}\right|^{2}\right\rangle \propto \exp \left\{-\frac{2 \kappa^{2}}{\rho^{-2}+\hat{L} k_{\perp}^{2}}\right\}
$$

where $\kappa=\omega /(c \sin \vartheta)$ is the incident frequency with $\vartheta$ the angle between input beam and density gradient (assuming slab geometry), $\rho$ is the beam width, and the expression $\hat{L} k_{\perp}^{2}$ is an average over the turbulent wavenumber and frequency spectrum that takes all spectral contributions along the beam path into account. For increasing $k_{\perp}, \kappa$ decreases slightly due to $1 / \sin \vartheta$ and $\hat{L} k_{\perp}^{2}$ decreases more strongly due to lower spectral power at small scales. Together, this increases the effect of the exponential term. In order to find the transition wavenumber, it would be necessary to evaluate $\hat{L} k_{\perp}^{2}$, which will be done in a later publication. Here, we just give a quick estimate: Eq. (20) in [22] states that $\hat{L} k_{\perp}^{2} \gtrsim \kappa^{2}$ if the turbulence level is "strong enough". Assuming this case for $\mathrm{X}$ mode, this means that the effect of the exponential is small. For the downscaled turbulence strength $(S \geq 10), \hat{L} k_{\perp}^{2}$ decreases and it is expected that the exponential term becomes relevant. In O mode, $\hat{L} k_{\perp}^{2} \ll \kappa^{2}$, and the exponential is always relevant. This analysis, when taken quantitatively, could also give an explanation for the different knee positions seen in $\mathrm{X}$ mode versus $\mathrm{O}$ mode.

\section{Conclusions}

After a long period of refinements in the GENE simulations, the data exchange, the fullwave simulations, improvements in the quality and quantity of the Doppler 
measurements, the profile and equilibrium, and the availability of both $\mathrm{O}$ and $\mathrm{X}$ mode measurements, we finally have good agreement between measured and simulated Doppler derived density fluctuation wavenumber spectra, with only a $25 \%$ discrepancy in the wavenumber axis, subject to further investigation. Non-linear theoretical analysis [22] could identify the fluctuation strength dependent transition wavenumber, above which the scattered power declines rapidly, giving rise to an apparent knee in the spectrum that is independent of the underlying density fluctuation spectrum's knee. It is planned to undertake a detailed analysis.

The X mode spectra are shown to be more non-linearly saturated than spectra from $\mathrm{O}$ mode measurements. A similar consequence of non-linear saturation was also found in the FT-2 tokamak synthetic Doppler reflectometry effort [5].

These results serve as another piece in the validation efforts for plasma turbulence simulations. The reproduction of the spectral shape forms an important link between turbulence simulations and comparison to measurements.

It is planned to continue this undertaking as new Doppler reflectometry measurements and accompanying turbulence simulations become available.

\section{Acknowledgments}

The GENE simulations were performed on the HPC-FF computer cluster in Jülich, Germany and on the BOB Linux cluster at RZG; the IPF-FD3D fullwave simulations were performed on hazelhen at HLRS Stuttgart. The authors acknowledge support by the state of Baden-Württemberg through bwHPC. This work was also partly performed within the framework of the Helmholtz Virtual Institute on Plasma Dynamical Processes and Turbulence Studies using Advanced Microwave Diagnostics and within the collaboration with the Max Planck Institute for Plasma Physics (IPP), Garching and Greifswald, Germany. This work has been carried out within the framework of the EUROfusion Consortium and has received funding from the Euratom research and training program 2014-2018 and 2019-2020 under grant agreement No 633053. The views and opinions expressed herein do not necessarily reflect those of the European Commission.

\section{References}

[1] Conway G D, Poli E, Happel T and the ASDEX Upgrade Team 2010 Plasma Fus. Res 5 S2005

[2] Lechte C, Conway G D, Görler T, Tröster C and the ASDEX Upgrade Team 2017 Plasma Phys. Contr. Fusion 59075006

[3] Stroth U, Navarro A B, Conway G D, Görler T, Happel T, Hennequin P, Lechte C, Manz P, Simon P, Biancalani A, Blanco E, Bottereau C, Clairet F, Coda S, Eibert T, Estrada T, Fasoli A, Guimarais L, Gürcan Ö, Huang Z, Jenko F, Kasparek W, Koenen C, Krämer-Flecken A, Manso M E, Medvedeva A, Molina D, Nikolaeva V, Plaum B, Porte L, Prisiazhniuk D, Ribeiro T, Scott B D, Siart U, Storelli A, Vermare L and Wolf S 2015 Nuclear Fusion 55083027 URL http://stacks.iop.org/0029-5515/55/i=8/a=083027

[4] Happel T, Görler T, Lechte C, Bernert M, Conway G D, Freethy S J, Hennequin P, Honoré C, Pinzón J, Stroth U and the ASDEX Upgrade Team 2017 Plasma Phys. Contr. Fusion 59 054009

[5] Krutkin O, Altukhov A, Gurchenko A, Gusakov E, Irzak M, Esipov L, Sidorov A, Chôné L, Kiviniemi T, Leerink S, Niskala P, Lechte C and Heuraux S 2019 Nucl. Fusion 59

[6] Kolmogorov A N 1941 Dokl. Akad. Nauk. SSSR 30 9-13 reprinted in Proc. R. Soc. Lond. A 434 (1991)

[7] Kraichnan R H 1967 Phys. of Fluids 101417

[8] Jenko F, Dorland W, Kotschenreuther M and Rogers B N 2000 Phys. of Plasmas 71904 
[9] Görler T, Lapillonne X, Brunner S, Dannert T, Jenko F, Merz F and Told D 2011 Journal of Computational Physics 230 7053-7071

[10] Lechte C 2009 IEEE Trans. Plasma Sci. 37

[11] Lechte C, Holzhauer E, Stroth U and Conway G 2007 Full Wave Doppler Reflectometry Simulations in 2D Proceedings of the 8th International Reflectometer Workshop in St. Petersburg, Russia URL https://www.aug.ipp.mpg.de/IRW/IRW8/papers/0810-IRW08LechteC_paper.pdf

[12] Taflove A and Hagness S C 2000 Computational Electrodynamics (685 Canton Street, Norwood, MA 02062: Artech House)

[13] Young J L 1994 Radio Science 29 1513-1522

[14] Xu L and Yuan N 2006 IEEE Antennas and Wireless Propagation Letters 5 335-338

[15] Yee K S 1966 IEEE Trans. Antennas and Propagation 14 302-307

[16] McCarthy P J, Martin P and Schneider W 1999 The CLISTE interpretive equilibrium code Tech. Rep. IPP Report 5/85 Max-Planck-Institute for Plasma Physics Garching, Germany

[17] Poli E et al. 2001 Comput. Phys. Commun. 139

[18] Lechte C 2018 URL https://doi.org/10.5281/zenodo.3696852

[19] Pinzón J R, Happel T, Blanco E, Conway G D, Estrada T and Stroth U 2016 Plasma Phys. Contr. Fusion 59035005

[20] Conway G D, Lechte C, Poli E, Maj O and the ASDEX Upgrade Team 2019 Recent progress in modelling the resolution and localization of Doppler reflectometry measurements Proceedings of the 14th International Reflectometry Workshop (IRW14) O.213 URL https://www.aug. ipp.mpg.de/IRW/IRW14/papers/213-IRW14-Conway-paper.pdf

[21] Gusakov E Z and Popov A Y 2004 Plasma Phys. Contr. Fusion 46 1393-1408

[22] Gusakov E, Surkov A and Popov A 2005 Plasma Phys. Contr. Fusion 47 